\title{
A Software Framework for the Automated Production of Schematic Maps
}

\author{
Joao Mourinho $^{1}$, Teresa Galvao ${ }^{1}$, João Falcão e Cunha ${ }^{1}$, \\ Fernando Vieira ${ }^{2}$, and Jose Pacheco ${ }^{2}$ \\ 1 Faculdade de Engenharia da Universidade do Porto, 4200-465 Porto \\ 2 OPT - Optimizacao e Planeamento de Transportes, 4200 Porto \\ joaomourinho@fe.up.pt
}

\begin{abstract}
Schematic Maps are mainly used for depicting transportation networks. They are generated through a schematization process where irrelevant details are eliminated and important details are emphasized. This process, being manually performed by teams of expert designers, is expensive and time consuming. Such manual execution is unsuitable for the production of schematic maps for location-based services or ondemand schematic maps, as near real-time and user-centered properties are needed. This work proposes GeneX, a framework that can support the automated generation of schematic maps. The framework and a new algorithms developed were able to completely eliminate erroneous map point placement, and to decrease by $33 \%$ the contention for map point placement, producing schematic maps without human intervention in soft real time.
\end{abstract}

Keywords: Schematic Maps, Software Framework, Public Transportation.

\section{Introduction}

Maps are part of the communicative process intended to communicate space information: the producer of maps (the sender) communicates to the receiver the message (map). The making of maps was only possible through the use of symbols and abstraction (which serve as a language), as maps are mainly intended to communicate space information. Schematic maps have been increasingly used in response to the need of better and simpler maps to describe complex transport networks, and they take the abstraction of space to a higher level, reducing the amount of information in comparison with traditional maps. This apparent simplicity is achieved through a simplification process called "schematization process" where choices are made regarding the level of detail and simplification. A special type of schematic map, called spider map, has also appeared recently. It presents innovative features such as a spider structure to improve visual presentation, user learning and spatial context communication. Schematic maps, by their inherent simplicity and symbolic meaning are good maps for being used in the transportation area as they are far more intuitive than conventional maps [1]. 
In fact as people travel more often, they need flexible and easy to understand maps which may take in account their context [2]. Automation in the production of maps is a key factor to achieve flexibility to tailor maps to user context, as it happens with Location-Based Services [3] where "information services accessible with mobile devices through the mobile network which have the ability to make use of the location of the mobile device" [4. There is the need, then, to develop a software framework which could support efficiently and comprehensively the automated generation and test of schematic maps. In this paper we propose and describe a software framework which serves as an engine to the automated generation and test of schematic maps. The structure of the paper is as follows. The main concepts regarding the definition of a schematic map and in particular, of a special type of context based schematic maps that we have called Spider Maps, are presented in Section 2. In Section 3 we present the related work concerning the schematization algorithms. In Section 4 we present the framework and discuss its development, architecture, and its enhancements. In section 5 we discuss the results obtained so far. Section 6 provides concluding remarks and clues about future work.

\section{Spider Maps}

\subsection{Schematic Maps}

Since the begining of the industrial revolution, the need of highly efficient, easily understandable transportation maps pushed the evolution of the traditional maps, and new forms of cartographic representation have emerged. Among the new forms of cartographic representation that have emerged, the schematic maps were probably the most bold. One remarkable schematic map applied to a transportation network was the Harry Beck's London Underground diagram, depicted in figure 2, As it is possible to observe, it presents radical changes with former London underground maps presented in figure 4.

Beck's map was considered both bold and innovative, as for the first time lines were drawn either horizontally, vertically or diagonally at 45 . This map also uses a non-linear scale, so the central area of the diagram is shown at a larger scale than the extremities. It shall be noted that although it does not mimic the geography of London, this map gives the traveler some clues about the terrain features (ex: river) and his/her location. Some authors define schematic map as "an easy-to-follow diagrammatic representation based on highly generalized lines which is in general used for showing routes of transportation systems, such as subways, trams and buses, or for any scenario in which streams of objects at nodes in a network play a role" [5]. The most important advantage of schematic maps is that they provide a quick view of the layout of the network by removing unimportant information like the detailed shape of the connections.

\subsection{Spider Maps}

Schematic spider maps are a special type of schematic maps. As with traditional schematic maps, the stops and lines of the transportation network correspond to 


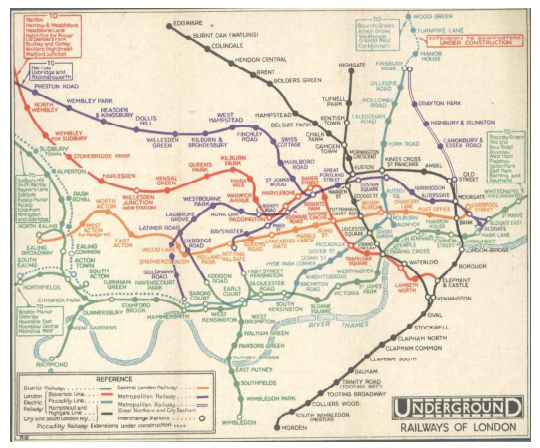

Fig. 1. London Underground Map prior to Beck's map. Source: http://www.chadsayshello.com

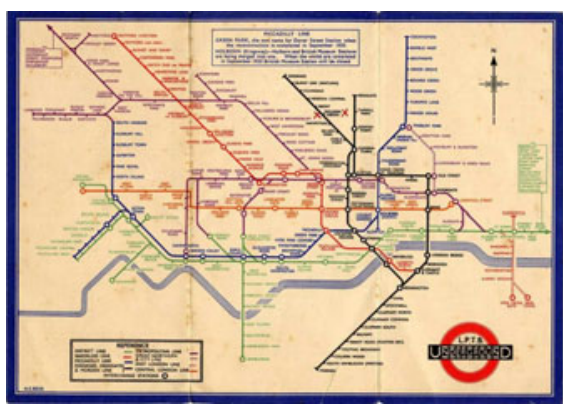

Fig. 2. Harry Beck's London underground map. Source: http://britton.disted.camosun.bc.ca

vertices and edges, respectively, in the spider map. However, they have enhanced features such a spider architecture, thus having a specific set of characteristics which sets them apart from the traditional schematic maps. Spider maps pay special attention to the context in order to enhance its learning by the users and it ease of use. While a schematic map is mainly used for the representation of metropolitan networks which remain fixed for several years, a spider map can be used (in London, for example) to represent bus transportation networks which, by its nature, are much more complex and unstable. In fact, if an underground transportation network can be represented with a single schematic map, it would not be feasible to represent a relatively complex bus transportation network using a single schematic map, due to the large number of bus stops and lines. To overcome this problem, spider maps allow the depiction of a schematic map for a particular geographic area. Hence, in order to represent a bus transportation network within a city, several spider maps can be produced (similar to the one depicted in Figure 10. The spider map architecture, depicted in figure 3 comprehends three main components:

- Hub: The hub is the main part of the map. Describes the area in which the user is, as well as the surrounding area with a higher degree of detail (buildings, roads, etc). The hub, as it is the central part of the spider map, is the first component the user will look at, as it makes uses of "focus and context" [6] and detail focusing [7] techniques. The hub is the only part of the spider map which does not comply with the 0/45/90 degrees line orientation. It can also include landmarks to allow the user to know important details about the place he currently is.

- Lines: The lines follow the orthogonal orientation of the traditional schematic maps, and describe the paths of the transport network where the user can go through while being at the zone depicted by the hub.

- Stops: The stops are the main destinations accessible to the user from the hub. 


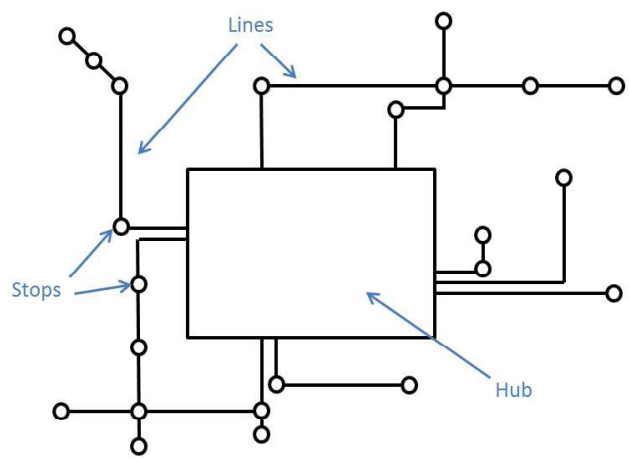

Fig. 3. Spider Map structure sketch

Figure 4 shows a spider map bus transportation network at a specific location, in this case, Victoria Station. It is possible to observe the spider map innovations in comparison with London underground maps of figures 1 and 2 .

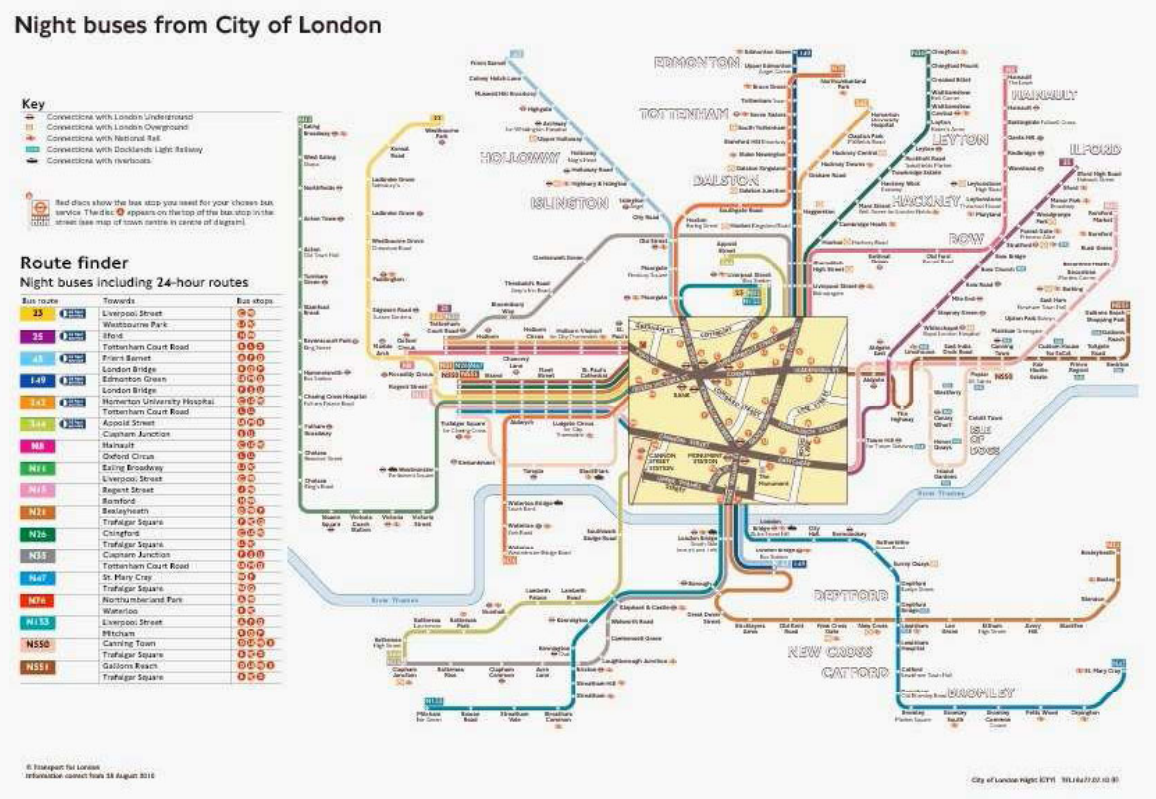

Fig. 4. London Bus Spider Map Source: http://www.tfl.gov.uk 


\section{Related Work}

Schematic maps have been increasingly used in response to the need of better and simpler maps to describe complex transport networks. This visual simplicity is achieved through a sequential decision process regarding the level and nature of detail and schematization. In practice, this "schematization process" is still a manual process carried away by teams of experts, such as designers and cartographers, despite the efforts in automating the process through the use of computers. The use of computers to execute the schematization process requires effective and efficient algorithms, to achieve in one hand high quality schematic maps which can be understood by people and, in the other hand, a time efficient process. Through schematization, certain map details are emphasized while others are deemphasized. It is fundamental, however, to present the smallest amount of information the user needs to learn the map: the more information presented, the higher the learning time will be. Latto defends that information shall be reduced to its basic components to achieve that goal [7].

There are some studies regarding research on the automated drawing of schematic maps. Some studies [8] 9] focus on the rectification of lines while others focus on the optimization of schematic maps [10. Barkowsky et al studied the application of the Discrete Curve Evolution to the schematization of maps [1]. Nevertheless these studies do not analyze the schematic map generation as a wide multidisciplinary problem as they tend to focus only on some areas of the problem. The automatic approaches for the generation of schematic maps mostly focus on the schematization process [12. Nollenburg [13] 14 makes an extensive research on the discrete mathematical foundations which are the basis of the algorithms used in the drawing of schematic maps and makes some brief considerations about their implementation. Nevertheless, his studies do not cover the human perception factors nor a concrete computer framework for drawing schematic maps. Silvana Avelar [1] 5] presents a wider study, by including some human perception factors and makes a complete research on the schematic maps on demand, one of the components to be integrated in the new paradigm. She goes further on by presenting a framework for electronic schematic maps which can answer user queries and studies the automated generation of schematic maps. Nevertheless, the study of the human perception factors is limited to what she calls the "aesthetic factors".

Most of the algorithms to design schematic maps retain a common structure [15]. They use a graph to model the transportation network, in which the vertices represent stops or turning points and the edges represent the paths between two turning points. In fact, the automated generation of schematic maps is truly a multidisciplinary problem, which involves integrating knowledge from several science fields. Isolated studies of different areas of knowledge, such as cognitive psychology show that user centered maps have a better performance and allow users to commit less errors [2]. Hochmair, for example [16] studied the effectiveness in the context on route planning, more specifically, as a measure of how well the map information supports the map reader in planning the fastest route between trip origin and destination on a public transportation map. 


\section{The GeneX Framework}

In this section, we present the GeneX framework which was developed through a collaboration research performed by a team involving collaborators from FEUP1, OPT 2 , STCP 3 , FWT4, INEGI 5 , and that was funded by INEGI.

\subsection{Overview}

The GeneX framework is a software application designed to support the following objectives:

1. The automatic generation of electronic schematic maps for complex transportation networks in bounded time, through the flexible use and parameterization of schematization algorithms

2. To serve as a test lab to support the research of schematic maps.

By merging and processing different kinds of external information (transportation networks, geographic/topological and constraint information) through the use of state-of-the-art algorithms, the framework generates schematic maps automaticaly. It is possible for the user to choose the desired algorithm and the parameters which will influence the map schematization. The framework produces an output SVG 6 (Scalable Vectorial Graphics) file which can be used directly in an electronic way, printed in paper or further processed (e.g. manual changes). The framework may also produce a statistics file which measures several parameters about the framework functioning.

\subsection{Software Engineering Life Cycle model}

The requirements elicitation and validation was performed together with the project stakeholders, as part of a Joint Application Development (JAD) model [17. The contributions provided by the partners were highly regarded: from the experts in information systems for transportation services (OPT) which provided real transport network data and expertise in automated solutions for transportation network systems, in optimization algorithms (INEGI) and map design (FWT), to the final users of the system (STCP). The development of this framework was considered, since the begining, an interdisciplinar subject in which knowledge from several areas of the science need to be integrated.

${ }^{1}$ Faculty of Engineering of University of Porto http://www.fe.up.pt

${ }^{2}$ OPT is an company based in Porto which develops IT infrastructures for Transportation Services. http://www.opt.pt

${ }^{3}$ STCP is a public transportation company operating in Porto. http://www.stcp.pt

${ }^{4} \mathrm{FWT}$ is a company based in London which produces maps for transportation networks. http://www.fwt.co.uk

${ }^{5}$ INEGI is a research institute based in Porto.

6 The Scalable Vectorial Graphic is XML language to describe vectorial bidimentional graphics. It is an open format created by the World Wide Web Consortium. 
Regarding functional requirements, the framework should be capable of producing schematic and spider maps in a fully automated way, about any location the user may select, using several schematization algorithms. The framework should be able to obtain data through the use of a common standard protocol data schema shared by the stakeholders. Another requirement was that the producton of schematic maps should be time-bounded (by setting deadlines or iterations number), to make it able to support Location-based services. In order to serve as a test lab, the framework should allow the choice of the algorithm and its parameters. The framework should also support different schematization algorithms (genetic, linear, tabu search, GRASP, etc) and provide a common protocol to implement them. This set of functional requirements needs to be supported by a set of non-funcional requirements, which comprehends usability, performance and interoperability. Usability is fundamental as the schematic maps produced by the framework have a strong user learning component: the maps produced, as well all possible interactions should be as intuitive as possible to be quickly learned and understood by their users. The designers and the final user team members provide insightful highlights in this area. To be able to support location-based services, the framework should execute the algorithm and produce the correspondent schematic map in near real time. Therefore, the framework was designed to perform as a soft real-time system [18]. The idea of a real-time approach is to answer queries within time constraints [18. One way of looking at a real-time system is through the metaphor of a stimulus/response system, where to each stimulus the system is supposed to produce an adequate response in an adequate time (deadline). If a specific system fails to produce a response to a stimulus until the deadline time and that means the value of the system is zero or negative (i.e. it causes cost, damage or negative profit), then that system is a hard real-time system. If a specific system fails to produce a response to a stimulus until the deadline and that means the value of the system is still positive after the deadline, then the system is a soft real-time system. This difference is depicted in figure 5 .

The figure depicting the hard real-time system shows two possible value functions after the deadline time, the red line depicting a system in which the failure to meet the deadline implies losses or damages (ex: safety critical system). As it is possible to observe, if there is any kind of tolerance after the deadline (i.e. the value, although may not be at its maximum value, is still positive), we have a real-time system. This is the case with the GeneX framework. Regarding interoperability, the framework should not only be compatible with the systems and data of the stakeholders, but it also should be able to support several different algorithms (every algorithm which conforms to a common implementation interface). Therefore, a standardized transport network data specificication was implemented and a common interface for the algorithms was designed. This was achieved making use of reusable components citeNeighbors1984 [19].

The framework was developed by using C\# Language, as a modern Object Oriented language which supported the requirement list. 

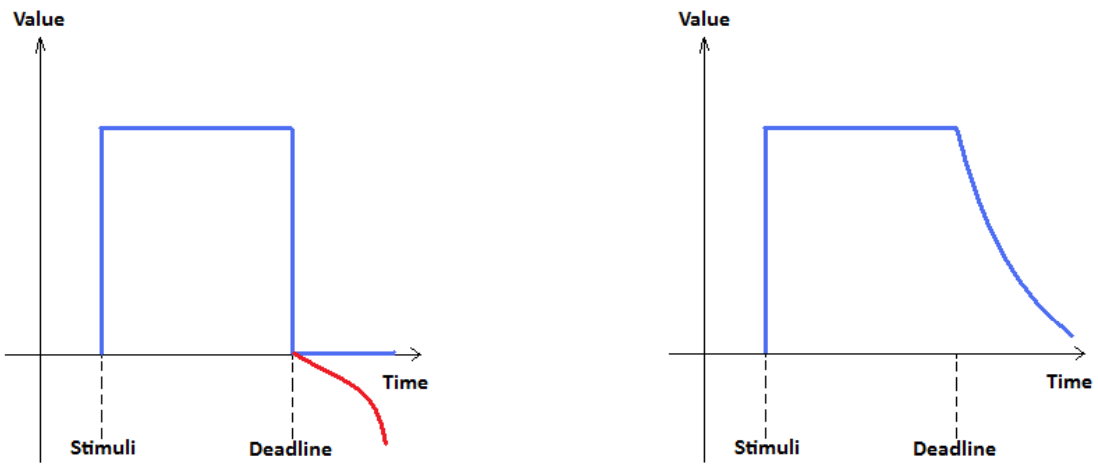

Fig. 5. Hard real-time system (left) and soft real-time system (right) stimulus/response metaphor

\subsection{Architecture and Data Model}

The architecture of the framework follows a modular structure, with two main modules: the data preparation module and the algorithm execution module. Figure [ shows the GeneX framwork package diagram, depicting its components.

The data aquisition and preparation module is responsible for preparing the data to be used by the algorithm execution module. The user selects graphically the location where he wants the spider/schematic map to be centered (hub).

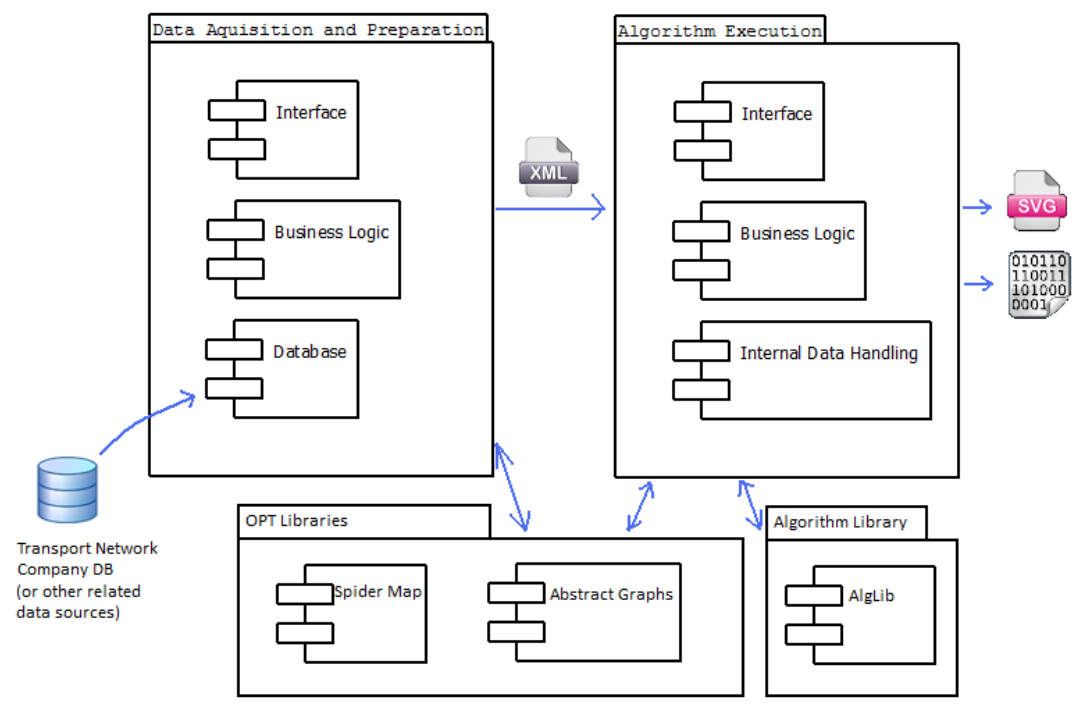

Fig. 6. GeneX Framework Package Diagram 
The module then extracts raw data from the transport network database and organizes it into a data structure by using the Spider Map Library. The spider map library is a complex set of $\mathrm{C} \#$ classes that support the serialization and communicaton of the spider and schematic map structure. Figure 7 depicts its class diagram.

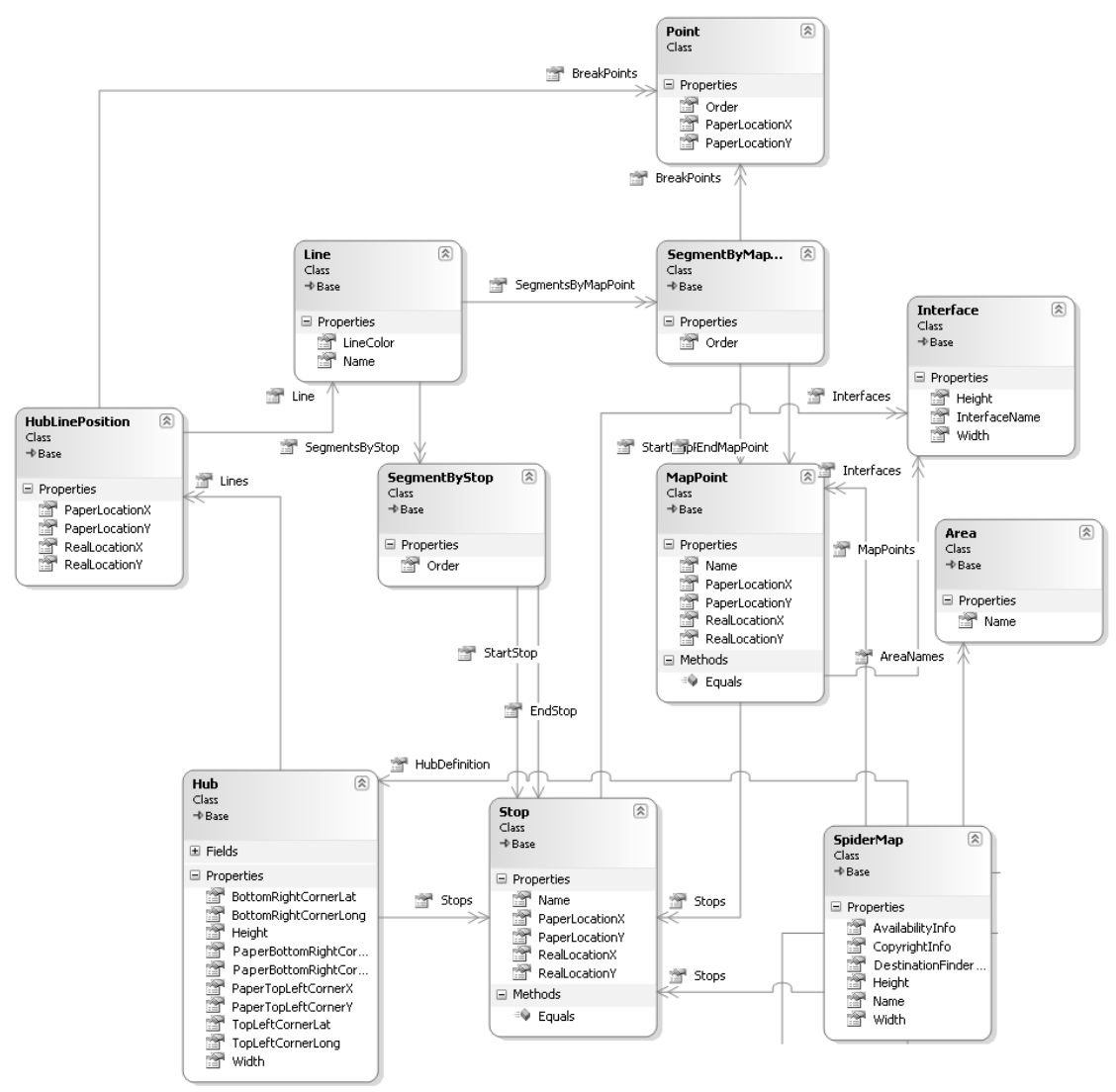

Fig. 7. Spider Map Library class diagram, showing the relations between the map components

The central class is the SpiderMap class, which contains attributes regarding the general properties of the map, such the name and dimensions. Each SpiderMap contains the definition of its hub in the Hub class. The definition of the hub encompasses its location and dimensions and also a set of HubLinePositions (which are the points where the lines start to flow from the hub to the map). The class HubLinePosition has information about its coordinates and the corresponding line (Line class). Each line may contain a set of ordered segments 
which have a MapPoint in each extremity. The mapPoint has location coordinates and has also a name. A line can be also a set of segments which have a Stop in each extremity. The difference between mapPoints and Stops is that a mapPoint usually depicts an certain important area made of a set of Stops. This is useful in real maps to depict very high density areas. This library is used to produce an XML file containing all the spider/schematic map data structure to be further processed by the algorithm execution module. The hierarchy of the XML file produced maps the components of the spider map according to table 1 .

Table 1. Transportation Network XML Data structures mappings

\begin{tabular}{|c||c|l|}
\hline Hierarchy \# & Structure & Transportation Network \\
\hline 0 & Map & Represents the whole Map. \\
1 & Hub & The central part of the map. Useful for Spider Maps. \\
2 & HubLinePos & The initial point of the transportation lines. \\
3 & Line & Each line of the transportation networks. \\
4 & Segment & Each line segment between two MapPoints. \\
5 & MapPoints & The base element. Can represent one or a set of stops. \\
\hline
\end{tabular}

The algorithm execution module reads the XML file and transforms the data into an internal data structure (to improve component reuse by the schematization algorithms). At the user interface, the user can choose the schematization algorithm (available at the AlgLib algorithms package) to execute and the corresponding options and performance measurement metrics. The business logic is then responsible for calling and executing the algorithm and to produce the final result, which can be an SVG (Scalable Vector Graphics) file (a standard vectorial format) or a binary file containing serialized data. The SVG file is produced by using a library that allows the conversion of a spider map data structure in an SVG file (Abstract Graphs Library) [20.

\subsection{The HPPO Algorithm}

The AlgLib package provides a foundation for the execution and configuration of the schematization algorithms. Each algorithm has to implement the same communication interface functions, in order to be used by the execution module:

- spiderMap execute(spiderMap, parameterList) performs the execution of the algorithm. The argumens are the spider map XML structure that was opened by the execution module, and the algorithm parameter list, already set up by the user. This function returns a spiderMap data structure which is the processed spider/schematic map. That structure can then be output to a SVG file by using Abstract Graphs library or serialized to a binary file. 
- parameterList getParameters() the module calls this parameter function to get the list of the algorithm parameters that can be set by the user, prior to the execution of the algorithm.

We have implemented a preliminary schematization algorithm that we called "Heuristic Point Placement Optimization" (HPPO). HPPO, aligns map points (corresponding to the transportation network stops and stations) to a regular grid, by positioning each map point in the nearest grid intersection. For high density or non regular density transportation maps, map station contention for grid intersections will happen. In this case, the HPPO smartly solves the contention through an heuristic algorithm. By using regular expressions, the point labels are also taken into account when placing network vertices, by determining the similarity degree of node labels in conjunction with its geographic location in order to produce a decision about the location to plot the node where the contention arises. The use of regular expressions is based in a finite-state automaton which scans strings (sets of letters in words) in order to find the degree of similarity. Therefore, if we have a node labeled "St. James Park" and other labeled "Park St. James", the automaton will tell us that those nodes refer, with a very high degree of certainty, the same node. So we are not only relying in geographical information, but also using computer science theory and cognitive psychology (to the preservation of topological relations has positive effects in the orientation and intuitiveness [21]) to make a higher quality judgement on how to solve the contentions that arise. For each map point, HPPO starts by checking if the nearest grid intersection is empty. If it is, then there is no contention and the point is plotted there. If the grid intersection is not empty, then we have a contention. In case there is a contention, that means that the geographical coordinates of the nodes are equal, or at least, within the same decision range concerning the square grid resolution. The automaton also tells us the degree of similarity. If the degree of similarity is higher than the predefined threshold level, then we assume that both nodes refer to the same location. In this case, they should be both plotted in the same grid intersection. If the degree of similarity is lower than the threshold, then it is important to distinguish them and plot them in different grid intersections while maintaining the topological relation between them. In this case, we get the topological relation between the two points (based on their coordinates) and try to move the node to the adequate grid intersection. If contention happens again (what can happen in a highly crowded map or with a loose square grid), then we have two options: or we may continue this cycle recursively until the topological relation is violated, or we may decide if we shall plot the node into the suggested grid intersection. To limit the processing time, we discarded the recursive approach in our algorithm. Being so, we analyse the proposed grid intersection. If contention happens we check the degree of label similarity through our automaton, and if is higher than the threshold, we plot the point there. If not, we analyse both the firse and the actual grid intersections suggested and we add the node where there are less nodes plotted. The pseudoalgorithm is described in figure 8. 


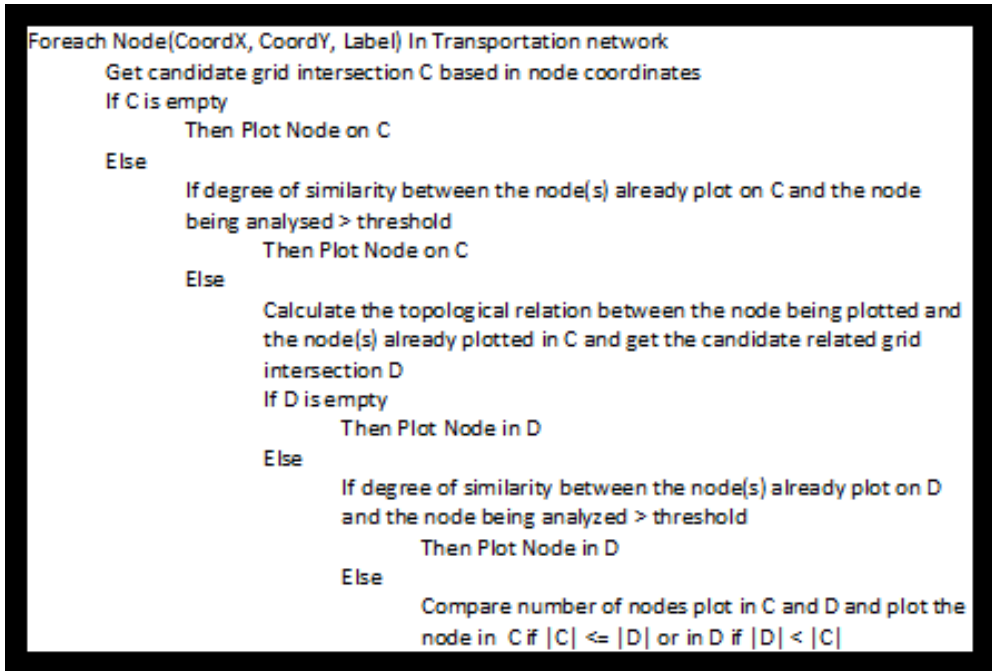

Fig. 8. HPPO pseudoalgorithm description

\section{Results}

The GeneX Framework is able to generate in a fully automated way schematic and spider maps for every location requested. It is also a very important tool as a test lab for the schematization algorithms that are being developed. Figure 9]

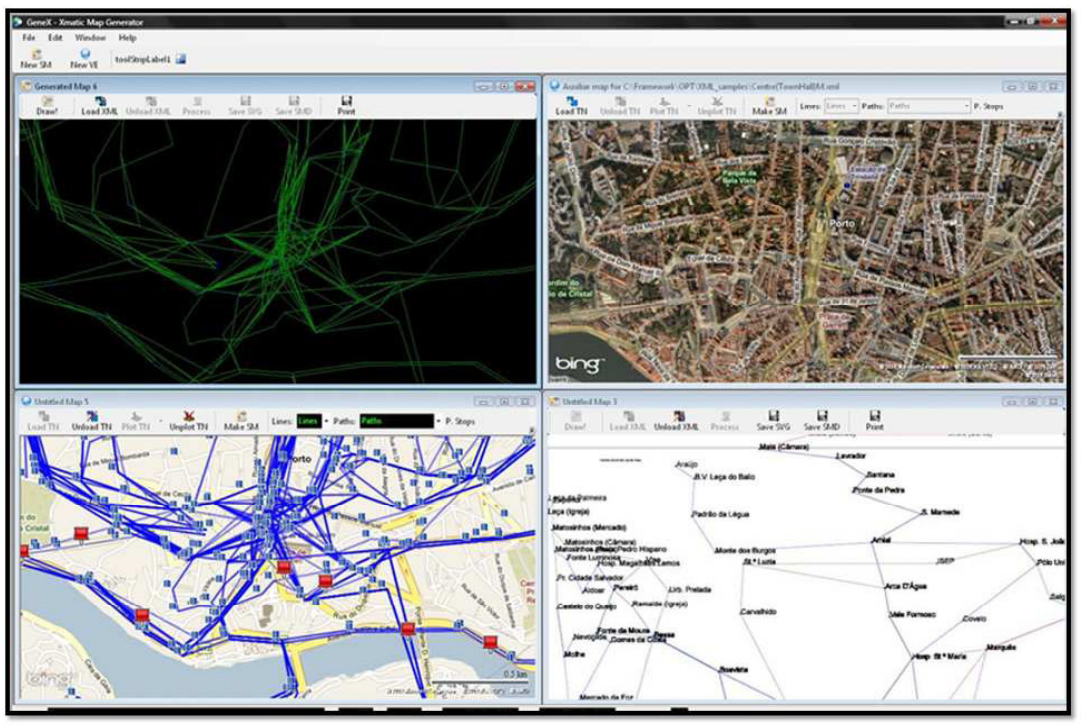

Fig. 9. User interface of the algorithm execution module, featuring an Multiple Document Interface showing several views about a schematic map 


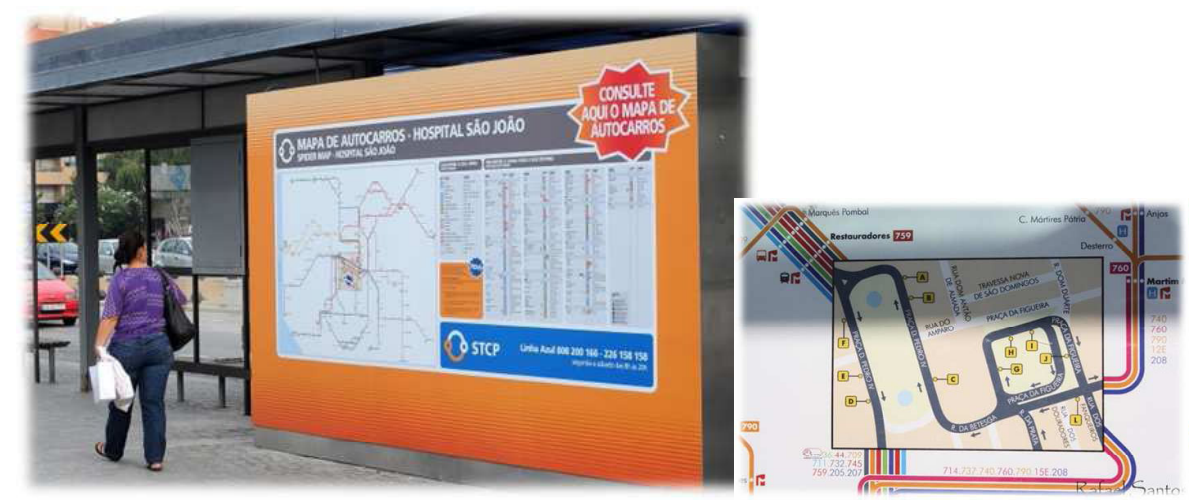

Fig. 10. Bus transportation network maps produced by using the GeneX Framework: city of Porto (left) Lisbon (right)

shows the user interface of the algorithm execution module, presenting multiple views of a map just before being processed by the schematization algortithms. It was found, in comparison with alternative approaches, that a multi-window environment presents a set of advantages to the generation of schematic maps, as it is much easier to assess the quality of the generated maps if we can compare side-by-side the schematization schema and the real map and geography of the terrain. This provides a valuable feedback for the tuning of the algorithms.

Although the framework is still under development and the algorithms in the AlgLib are being improved and enhanced, it is already being used for the production of schematic and spider maps. Maps produced with this framework are already available for public use in the cities of Porto and Lisbon, as it is possible to see in figure 10. As the schematization algorithms currently in use are still being perfected, those maps were also subjected to manual intervention to improve their aesthetical appearance.

Concerning our HPPO algorithm, it showed good results as can be shown at figure 11; it was shown that its use reduces by $33 \%$ the number of grid intersections that have dissimilar nodes plotted ("Bad Cells"). Other advantage of HPPO is that if different nodes refer to the same place, this algorithm can ignore contention and plots them correctly in the same grid intersection. Another advantage is that this algorithm, due to the use of regular expressions can identify nodes with similar labels which refer to the same location, grouping them in the same node. In addition, all the topological relations are still preserved. For a map with 45 MapPoints/Stops, it takes 2 seconds to execute, which is in compliance with the soft realtime requirements. Nevertheless, the decrease of $33 \%$ in the number of bad cells may not be good enough for evenly distributed very high density maps with a great number of mapPoints, as this algorithm performs better in maps which have fluctuations in mapPoint density. In the former case, its 

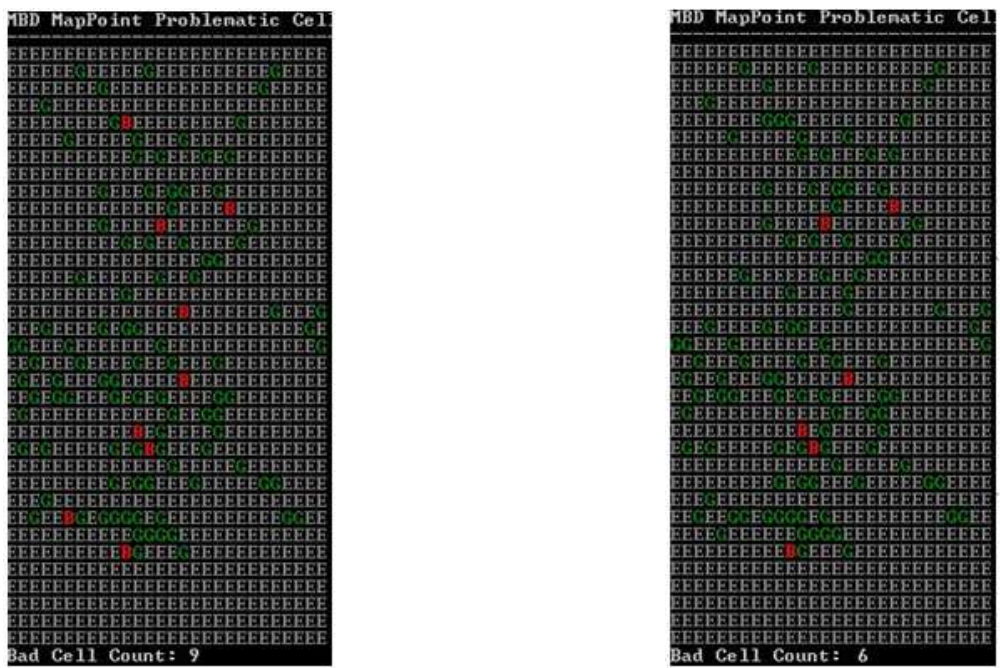

Fig. 11. Frequency matrixes, depicting the transport network grid intersections with ASCII characters, obtained after the execution of the algorithm. It shows Bad Cells (in red) when not using $\mathrm{HPPO}$ (left) and using $\mathrm{HPPO}$ (right) for the Porto downtown spider map. HPPO reduced Bad Cells from 9 no 6, while preserving topological relations.

effectiveness can be reduced while in the later case its effectiveness can be enhanced. Therefore, this algorithm does not guarantees that the generated schematic maps contains no bad cells, so there is still margin for further improvement.

\section{Conclusions and Future Work}

The GeneX framework was used to produce real spider maps that are being used in the cities Porto and Lisbon, it has proved effective in generating spider maps. Altough these maps were produced by an automated process, they were subjected to small manual changes to improve the visual appearance, which is the most difficult aspect to model in the algorithms. For simpler maps with less lines and stops, the framework is able to generate them with good results. The quality of the results of the HPPO algorithm is quite good, showing a significant decrease in bad cells. The algorithms need to be perfected in order to increase the quality of the results and to make them directly usable (without any manual processing). The algorithms need to further improve aspects such as visual line distiction, stop label organization and geographical constraints. Some development of the framework is also needed to support Location-based services, such a "request manager" which can feed user requests to the framework and reply to them. Other issues that need further study are the adaptation of the resulting maps to different devices. All this work also needs to be complemented and validated with usability tests and analysis. 


\section{References}

1. Avelar, S.: Schematic Maps on Demand: Design, Modeling and Visualization. PhD thesis, Swiss Federal Institute of Technology Zurich (2002)

2. Porathe, T.: User-Centered Map Design. Design (2007)

3. Steiniger, S., Neun, M., Edwardes, A.: Foundations of location based services (2006)

4. Virrantaus, K., Markkula, J., Garmash, A., Terziyan, Y., Veijalainen, J., Katanosov, A., Tirri, H.: Developing GIS-supported location-based services. In: Proc. of WGIS, pp. 423-432 (2001)

5. Avelar, S., Hurni, L.: On the Design of Schematic Transport Maps. Cartographica: The International Journal for Geographic Information and Geovisualization 41, 217-228 (2006)

6. Bogen, S., Brandes, U., Ziezold, H.: Visual Navigation with Schematic Maps. In: Visual Information Communication, pp. 65-84 (2010)

7. Latto, R.: Do we like What We See? (2004)

8. Cabello, S., Deberg, M., Vankreveld, M.: Schematization of networks. Computational Geometry 30, 223-238 (2005)

9. Cabello, S., Kreveld, M.V., Sciences, C., Box, P.O.: Schematic Networks: An Algorithm and its Implementation. In: Richardson, D., Oosterom, P. (eds.) 10th International Symposium on Spatial Data Handling (SDH), Ottawa, pp. 475-486. Springer (2002)

10. Anand, S., Avelar, S., Ware, J.M., Jackson, M.: Automated schematic map production using simulated annealing and gradient descent approaches. Technology (2000)

11. Barkowsky, T., Latecki, L.J., Richter, K.F.: Schematizing Maps: Simplification of Geographic. Cognition 8, 41-53 (2000)

12. Ware, J.M., Taylor, G.E., Thomas, N.: Automated Production of Schematic Maps for Mobile Applications. Transactions in GIS 10, 25-42 (2006)

13. Nöllenburg, M.: Automated drawing of metro maps. PhD thesis, Universitat Karlsruhe (2005)

14. Nöllenburg, M., Wolff, A.: A Mixed-Integer Program for Drawing High-Quality Metro Maps. In: Healy, P., Nikolov, N.S. (eds.) GD 2005. LNCS, vol. 3843, pp. 321-333. Springer, Heidelberg (2006)

15. Dong, W., Guo, Q., Liu, J.: Schematic road network map progressive generalization based on multiple constraints. Geo-spatial Information Science 11, 215-220 (2008)

16. Hochmair, H.: The Influence of Map Design on Route Choice from Public Transportation Maps in Urban Areas. The Cartographic Journal 46, 242-256 (2009)

17. Scacchi, W.: Process Models in Software Engineering, pp. 1-24 (October 2001)

18. Laurini, R., Servigne, S., Nol, G.: Soft real-time GIS for disaster monitoring, pp. 465-480. Springer (2005)

19. Goguen, J.A.: Reusing and interconnecting software components. Computer 19, 16-27 (1986)

20. OPT: Abstract Graphs Library Specification, Internal Report (2009)

21. Casakin, H., Barkowsky, T., Klippel, A., Freksa, C.: Schematic Maps as Wayfinding Aids. In: Habel, C., Brauer, W., Freksa, C., Wender, K.F. (eds.) Spatial Cognition II. LNCS (LNAI), vol. 1849, pp. 54-71. Springer, Heidelberg (2000) 